\title{
Pronóstico de pacientes con lupus eritematoso generalizado en una unidad de cuidados intensivos
}

Silvio Namendys-Silva, Miguel Reyes-Ruiz, Eduardo Rivero-Sigarroa y Guillermo Domínguez Cherit

Secretaría de Salud, Instituto Nacional de Ciencias Médicas y Nutrición "Salvador Zubirán", Departamento de Terapia Intensiva, Ciudad de México, México

\begin{abstract}
Resumen
Introducción: La identificación de factores de riesgo para mortalidad ha permitido mejorar el tratamiento de los pacientes críticos con lupus eritematosos generalizado. Objetivo: Describir las características clínicas y los pronósticos de pacientes graves con lupus eritematosos generalizado ingresados en una unidad de cuidados intensivos. Método: Estudio de cohorte, observacional y descriptivo. Se incluyeron 207 pacientes con lupus eritematosos generalizado enero de 2011 y enero de 2016. Resultados: Durante el periodo del estudio fueron ingresados 3215 pacientes graves a una unidad de cuidados intensivos, 207 (6.4 \%) con lupus eritematosos generalizado después de ser evaluados por un intensivista. La mortalidad fue de $18.4 \%$. El análisis multivariado identificó la presencia fallas orgánicas (cardiovascular, hepática y neurológica) y niveles séricos de lactato $>2 \mathrm{mmol} / \mathrm{L}$ al ingreso o durante las primeras 24 horas de estancia en la unidad de cuidados intensivos como los principales factores asociados con incremento de la mortalidad. Conclusiones: Un dato relevante fue que la falla neurológica y la falla hepática estuvieron asociadas con mayor riesgo de muerte a 28 días, sin que identificáramos reportes similares a estos hallazgos.
\end{abstract}

PALABRAS CLAVE: Lupus. Síndrome antifosfolípido. Cuidados intensivos.

\begin{abstract}
Introduction: Identification of risk factors for mortality has enabled improving the treatment of critically ill patients with systemic lupus erythematosus. Objective: To describe clinical characteristics and prognoses of critically ill patients with systemic lupus erythematosus admitted to an intensive care unit. Method: Prospective, observational cohort study. A total of 207 patients with systemic lupus erythematosus admitted between January 2011 and January 2016 were included. Results: During the study period, 3,215 critically ill patients were admitted to the intensive care unit, out of which 207 (6.4\%) were identified as having systemic lupus erythematosus after being evaluated by an intensivist. The multivariate analysis identified the presence of organ failure (cardiovascular, liver, neurological) and serum lactate levels $>2 \mathrm{mmoL} / \mathrm{L}$ at admission or within the first 24 hours of intensive care unit stay as the main factors associated with increased mortality. Conclusions: $A$ relevant observation was that neurological failure and liver failure were associated with higher mortality risk at 28 days, without reports similar to these findings being identified.
\end{abstract}

KEY WORDS: Lupus. Antiphospholipid syndrome. Intensive care.

Correspondencia:

Silvio Ñamendys-Silva

E-mail: snamendys@medicasur.org.mx
Fecha de recepción: 30-07-2017

Fecha de aceptación: 18-01-2018

DOI://dx.doi.org/10.24875/GMM.18003614
Gac Med Mex. 2018;154:468-472

Disponible en PubMed www.gacetamedicademexico.com 


\section{Introducción}

El lupus eritematoso generalizado (LEG) es una enfermedad polimórfica reumatológica en la que existe autoinmunidad en todos los órganos y se caracteriza por exacerbaciones y remisiones. ${ }^{1}$ Es una patología proinflamatoria en la que el paciente está predispuesto a infecciones durante los estados de agudización, lo que puede ocasionar condiciones clínicas que ponen en peligro la vida. ${ }^{2,3}$ Por otro lado, el tratamiento inmunosupresor puede incrementar el riesgo de complicaciones sistémicas. ${ }^{4}$

Aunque no existen datos estadísticos precisos, la prevalencia del LEG es alta a nivel mundial, con tendencia al incremento. ${ }^{5,6} \mathrm{~A}$ pesar de lo anterior, la mortalidad ha disminuido en las últimas décadas, probablemente debido al diagnóstico más temprano y a la mayor disponibilidad de tratamientos inmunosupresores. $^{7-9}$ En los pacientes graves con LEG que requieren internamiento en una unidad de cuidados intensivos (UCl), la falla cardiovascular sigue siendo uno de los principales factores de riesgo para muerte. ${ }^{10,11}$ En algunas series se han identificado los siguientes factores predictores de muerte en pacientes críticos con LEG: infecciones del sistema respiratorio, puntuación alta de APACHE II, necesidad de terapia de sustitución renal, uso de ventilación mecánica y puntuación alta de SLEDAI.12,13 La identificación de factores de riesgo para mortalidad ha permitido mejorar el tratamiento de los pacientes críticos con LEG. ${ }^{14,15}$ El objetivo del presente trabajo fue describir las características clínicas y el pronóstico de pacientes graves con LEG admitidos en una UCl.

\section{Método}

Estudio observacional, descriptivo y analítico realizado en la UCl del Instituto Nacional de Ciencias Médicas y Nutrición "Salvador Zubirán", entre enero de 2011 y enero de 2016. La UCl dispone de 14 camas para atender pacientes graves y tiene la capacidad de brindar soporte avanzado como ventilación mecánica invasiva, hemodiálisis y plamaféresis. Se incluyeron todos los pacientes graves con LEG, de uno y otro sexo, mayores de 16 años, que permanecieron en la UCI por más de 24 horas. Los datos demográficos y clínicos fueron obtenidos del expediente clínico y del expediente electrónico. Los pacientes cumplían criterios para $L E G$ a su ingreso a la $\mathrm{UCl}$ o se clasificaron como LEG durante su estancia en la UCl.
Se realizó registro de los siguientes datos: edad, sexo, tiempo de diagnóstico de LEG, dosis de esteroides al momento del ingreso a la $\mathrm{UCl}$, diagnóstico de ingreso a la unidad, días de estancia en ella, lugar de procedencia, puntuación APACHE ${ }^{116}$ y puntuación SOFA $^{17}$ al ingreso a UCl, así como número de fallas orgánicas definidas por SOFA. ${ }^{18}$ Además, se registró la necesidad de vasopresores, dosis requerida, lactato sérico al ingreso, $\mathrm{PaO}_{2} / \mathrm{FiO}_{2}$ al ingreso a $\mathrm{UCl}$, necesidad de ventilación mecánica (VM) y motivo, días de VM, nivel de creatinina, conteo plaquetario y niveles de billirubinas. Asimismo, se registró la presencia de síndrome antifosfolípidos secundario (SAF) clínico o serológico y puntuación en la Escala de Coma Glasgow (ECG) antes de recibir sedación, de haberla requerido. ${ }^{16-20}$ Se registró la mortalidad a los siete y 28 días del egreso de la UCl.

\section{Análisis estadístico}

Se utilizó estadística descriptiva para la presentación de los datos y se realizó prueba de Kolmogorov-Smirnov para determinar su distribución. Las variables numéricas se expresaron como mediana y rangos intercuartílicos, dado que todas las variables continuas presentaron distribución no paramétrica; las variables nominales se representaron en número y porcentaje.

Se realizó análisis de regresión de Cox univariado y multivariado para identificar los factores relacionados con incremento de la mortalidad a 28 días. Las variables con relevancia clínica y las predictivas del punto final primario en el análisis univariado se introdujeron en el modelo final. Los resultados se expresaron como hazard ratios con sus respectivos intervalos de confianza de $95 \%$. Se estimaron curvas de supervivencia mediante el método de Kaplan-Meier. Los datos de supervivencia se compararon con la prueba de rangos logarítmicos (log-rank test). En todos los casos, una $p<0.05$ fue considerada estadísticamente significativa. Para el procesamiento de los datos se utilizó el programa estadístico SPSS 22.0.

El presente estudio se apegó a las consideraciones formuladas en la Declaración de Helsinki y su modificación de Tokio para los trabajos de investigación biomédica en sujetos humanos, así como a las consideraciones respecto a la investigación para la salud formuladas en la Ley General de Salud de los Estados Unidos Mexicanos. Por ser un estudio observacional no fue necesario el consentimiento informado por escrito de los pacientes. 


\section{Resultados}

Durante el periodo del estudio fueron ingresados 3215 pacientes graves a la UCl, entre ellos 207 (6.4\%) con LEG después de ser evaluados por un intensivista. La mediana de edad fue de 31 años y $88.9 \%$ fue del sexo femenino. La mediana del tiempo de diagnóstico de LEG fue de siete años. Las características generales de los pacientes incluidos en el estudio se muestran en la Tabla 1.

El $74.4 \%$ de los pacientes ingresó por un problema médico: infeccioso (46.4\%), de índole cardiovascular $(16.9 \%)$ y neurológica $(10.5 \%)$. Las fallas orgánicas que se observaron con mayor frecuencia fueron renales $(71.5 \%)$, hematológicas $(64.3 \%)$ y respiratorias (62.8\%). El $77.8 \%$ de los pacientes requirió VM; la media de la duración fue seis días. La mortalidad fue de $18.4 \%$ (38/207). La mortalidad de los pacientes con cuatro o más fallas orgánicas fue de $39.7 \%$ (29/73). En la Tabla 2 se observa la relación entre el número de fallas orgánicas y la mortalidad a 28 días.

De los pacientes que presentaron falla respiratoria, $20(9.7 \%)$ tuvieron diagnóstico de hemorragia pulmonar difusa (HPD) y 14 cumplieron con criterios de síndrome de insuficiencia respiratoria aguda (SIRA) $(6.8 \%)$. La mortalidad a 28 días de los pacientes con falla respiratoria fue de $28.6 \%$ (4/20).

En las Tablas 3 y 4 se detallan los resultados de los análisis univariado y multivariado para identificar los factores asociados con incremento de la mortalidad a 28 días. Los factores asociados con incremento de la mortalidad a 28 días fueron falla cardiovascular ( $H R=3.27, I C \quad 95 \%=1.41-7.54, p=0.005)$, falla hepática $(\mathrm{HR}=2.16, \mathrm{IC} 95 \%=1.11-4.22, \mathrm{p}=0.024)$, falla neurológica $(H R=3.42$, IC $95 \%=1.75-6-68$, $\mathrm{p}<0.001)$ y niveles séricos de lactato $(H R=1.17$, IC $95 \%=1.06-1.29, p=0.002)$ (Tabla 4 y Figura 1 ).

\section{Discusión}

Los principales hallazgos del presente estudio fueron:

- La mortalidad de pacientes graves con LEG ingresados a la UCl fue de $18.4 \%$.

- Los principales factores asociados con incremento de la mortalidad a 28 días fueron las fallas neurológicas, cardiovasculares y hepáticas.

- Los niveles del lactato al ingreso a la UCI o durante las primeras 24 horas de estancia constituyen un biomarcador de riesgo para muerte en pacientes graves con LEG.
Tabla 1. Características generales de 207 pacientes con lupus eritematoso generalizado ingresados en una unidad de cuidados intensivos

\begin{tabular}{|c|c|c|}
\hline Variable & $\mathrm{n}$ & $\%$ \\
\hline \multicolumn{3}{|l|}{ Sexo } \\
\hline Mujer & 184 & (88.9) \\
\hline Hombre & 23 & $(11.1)$ \\
\hline \multicolumn{3}{|l|}{ Procedencia } \\
\hline Urgencias & 160 & $(77.3)$ \\
\hline Hospitalización & 47 & $(22.7)$ \\
\hline \multirow[t]{2}{*}{ Mortalidad a 28 días } & 38 & $(18.4)$ \\
\hline & Mediana & RI \\
\hline Edad (años) & 31 & $24-43$ \\
\hline APACHE II (puntos) & 19 & $(14-24)$ \\
\hline SOFA (puntos) & 8 & $(6-11)$ \\
\hline Tiempo del diagnóstico (años) & 7 & $(2-13)$ \\
\hline Dosis de prednisona (mg/día) & 16 & $(5-50)$ \\
\hline Días de estancia en UCl & 4.5 & $(3-10)$ \\
\hline Lactato (mmol/L) & 2.0 & $(1.2-2.9)$ \\
\hline $\mathrm{PaO}_{2} / \mathrm{FiO}_{2}, \mathrm{~mm} \mathrm{Hg}$ & 180 & $(120-260)$ \\
\hline Creatinina (mg/dL) & 2.0 & $(1-4.8)$ \\
\hline Bilirrubina total (mg/dL) & 1.21 & $(0.9 .1)$ \\
\hline Plaquetas $\times 10^{3} / \mathrm{mm}^{3}$ & 112 & $(57-112)$ \\
\hline
\end{tabular}

Tabla 2. Relación entre el número de fallas orgánicas y mortalidad a 28 días en pacientes con lupus eritematoso generalizado ingresados en una unidad de cuidados intensivos

\begin{tabular}{lccccc}
\hline Fallas & & & & \multicolumn{2}{c}{ Mortalidad } \\
\cline { 6 - 6 } Número & $\mathbf{n}$ & $\%$ & & $\mathbf{n}$ & $\%$ \\
\hline 0 & 2 & 1 & 0 & 0 \\
1 & 34 & 16.4 & 1 & 2.9 \\
2 & 41 & 19.8 & 1 & 2.4 \\
3 & 57 & 27.5 & 7 & 12.3 \\
4 & 46 & 22.2 & 13 & 28.2 \\
5 & 23 & 11.1 & 14 & 60.8 \\
6 & 4 & 1.9 & 2 & 50 \\
\hline
\end{tabular}

Diferentes grupos han reportado disminución de la mortalidad de los pacientes con LEG a lo largo de la última década, lo que puede estar relacionado con mejoría en el tratamiento del LEG y a los avances tecnológicos en los cuidados intensivos, además de que se ha promovido a nivel internacional el ingreso temprano a la $\mathrm{UCl}$ con el menor número de fallas 

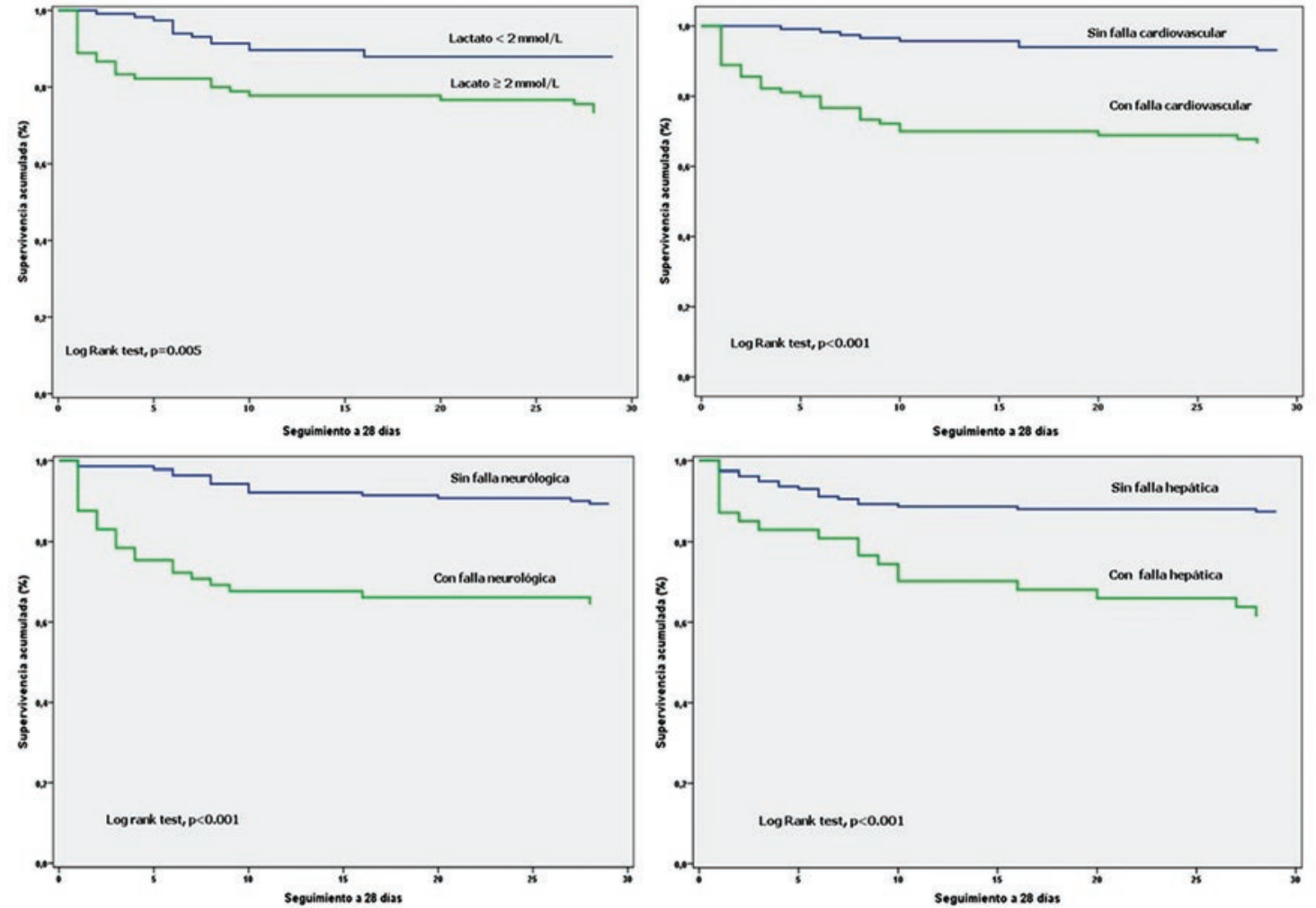

Figura 1. Curvas de supervivencias según los factores relacionados con la mortalidad a 28 días en pacientes con lupus eritematoso generalizado ingresados en una unidad de cuidados intensivos.

Tabla 3. Análisis univariado para identificar factores relacionados con mortalidad a 28 días en pacientes con lupus eritematoso generalizado ingresados en una unidad de cuidados intensivos

\begin{tabular}{lccc}
\hline Variable & HR & IC $95 \%$ & $\mathbf{p}$ \\
\hline Edad (años) & 1.03 & $1.01-1.06$ & 0.001 \\
Sexo masculino & 0.41 & $0.10-1.73$ & 0.230 \\
APACHE II & 1.11 & $1.07-1.16$ & $<0.001$ \\
SOFA & 1.37 & $1.25-1.51$ & $<0.001$ \\
Falla cardiovascular & 5.8 & $2.66-12.6$ & $<0.001$ \\
Lactato (mmol/L) & 1.28 & $1.17-1.39$ & $<0.001$ \\
Lactato > 2 mmol/L & 2.45 & $1.27-4.75$ & 0.008 \\
Falla respiratoria & 1.69 & $0.82-3.48$ & 0.154 \\
PaO/FiO ${ }_{2}$ (mmHg) & 0.99 & $0.99-1.0$ & 0.104 \\
Falla renal & 1.12 & $0.54-2.32$ & 0.741 \\
Falla hematológica & 2.66 & $1.17-6.04$ & 0.019 \\
Falla hepática & 3.44 & $1.82-6.52$ & $<0.001$ \\
Falla neurológica & 4.07 & $2.12-7.81$ & $<0.001$ \\
SAF & 0.72 & $0.35-1.46$ & 0.367 \\
SIRA & 1.62 & $0.57-4.57$ & 0.360 \\
HPD & 0.48 & $0.11-2.0$ & 0.317 \\
\hline
\end{tabular}

$\mathrm{HR}=$ Hazard ratio, $\mathrm{APACHE}=$ Acute Physiology and Chronic Health Evaluation, SOFA = The Sequential Organ Failure Assessment, SAF = Síndrome antifosfolipido, $\mathrm{SIRA}=$ síndrome de insuficiencia respiratoria aguda, $\mathrm{HPD}=$ Hemorragia pulmonar difusa.
Tabla 4. Análisis multivariado para identificar factores relacionados con mortalidad a 28 días en pacientes con lupus eritematoso generalizado ingresados en una unidad de cuidados intensivos

\begin{tabular}{lccc}
\hline Variable & HR & IC 95 \% & p \\
\hline Falla cardiovascular & 3.27 & $1.41-7.54$ & 0.005 \\
Falla hepática & 2.16 & $1.11-4.22$ & 0.024 \\
Falla neurológica & 3.42 & $1.75-6-68$ & $<0.001$ \\
Lactato $>$ 2 mmol/L & 1.17 & $1.06-1.29$ & 0.002 \\
\hline HR $=$ Hazard ratio. & & &
\end{tabular}

orgánicas. La mortalidad global en el presente trabajo fue menor a la reportada por otros centros. ${ }^{13,21}$ Dumas et al. ${ }^{11}$ reportaron una mortalidad de $20 \%$ en pacientes reumatológico, similar a la encontrada en el presente trabajo.

Las puntuaciones de APACHE II y SOFA ${ }^{16-19}$ han sido relacionadas con mayor mortalidad en pacientes graves con LEG. Nuestro grupo ${ }^{10}$ reportó que la puntuación del APACHE II fue de 19.7 durante las primeras 24 horas de estancia en la $\mathrm{UCl}$, similar al del presente reporte. Otros autores han informado puntuaciones de APACHE II mayores y mayor mortalidad. ${ }^{13}$

Pocos estudios han utilizado la puntuación SOFA para determinar si un paciente con LEG ingresa o no 
a la UCl; en el presente trabajo fue incluida y se identificó como un factor de riesgo para muerte. Otros autores, ${ }^{11}$ a pesar de no categorizar las puntuaciones de SOFA por fallas orgánicas, han reportado menor puntuación en comparación con la puntuación en nuestro grupo de pacientes con LEG (cinco versus ocho puntos), lo que sugiere que en la $\mathrm{UCl}$ de nuestro centro se aceptan pacientes con mayor número de fallas orgánicas o mayor gravedad, sin embargo, a pesar de esta diferencia en la puntuación de SOFA, la mortalidad fue similar ( $21 \%$ versus $18.3 \%$ ).

\section{Conclusiones}

Al evaluar el número y tipo de fallas orgánicas con los que ingresaron los pacientes, se encontraron resultados similares a los de otros estudios, ${ }^{10-13}$ siendo la falla cardiovascular uno de los principales factores de riesgo para muerte. Aunque en nuestro grupo de pacientes la falla orgánica con mayor prevalencia fue la renal, no tuvo impacto en la mortalidad. Un dato relevante fue que la falla neurológica y la falla hepática estuvieron asociadas con mayor riesgo de muerte a 28 días; en este sentido no hay reportes similares a nuestros resultados. Las alteraciones neurológicas de pacientes graves con sepsis, los eventos vasculares o estados epilépticos fueron las principales causas de afección neurológica en nuestra cohorte..$^{22,23}$ Se ha descrito que los pacientes con LEG y alteración neurológica tienen alta mortali$\mathrm{dad},{ }^{24}$ por lo anterior requieren vigilancia en áreas monitorizadas para mejorar el pronóstico. Finalmente, similar a lo reportado por otros autores, ${ }^{25,26}$ el nivel de lactato sérico durante las primeras 24 horas de estancia en la $\mathrm{UCl}$ se asoció con mayor mortalidad.

La principal limitante del presente estudio estribó en que fue realizado en un solo centro de alta concentración de pacientes con enfermedades inmunológicas.

\section{Bibliografía}

1. David P, Munther A, Graham R. Systemic lupus erythematosus. Lancet. 2007;369:587-596.

2. Navarra S, Leynes M. Infections in systemic lupus erythematosus. Lupus. 2010;19:1419-1424

3. Han BK, Bhatia R, Traisak P, Hunter K, Schorr C, Eid H, et al. Clinical presentations and outcomes of systemic lupus erythematosus patients with infection admitted to the intensive care unit. Lupus. 2013;22:690-696.
4. Ruiz-Irastorza G, Olivares N, Ruiz-Arruza I, Martinez-Berriotxoa A, Egurbide MV, Aguirre C, et al. Predictors of major infections in systemic lupus erythematosus. Arthritis Res Ther. 2009;11:R109.

5. Pons-Estel GJ, Alarcón GS, Scofield L, Scofield L, Reinlib L, Cooper GS. Understanding the epidemiology and progression of Systemic Lupus Erythematosus. Semin Arthritis Rheum. 2010;39:257-268.

6. Danchemko N, Satia JA, Anthony MS. Epidemiology of systemic lupus erythematosus: a comparation of worldwide disease burden. Lupus. 2006;15:308-318.

7. Souza DC, Santo AH, Sato El. Mortality profile related to systemic lupus erythematous: a multiple cause-of-death analysis. J Rheumatol. 2012;39:496-503.

8. Fei Y, Shi X, Gan F, Li X, Zhang W, Li M, et al. Death causes and pathogens analysis of systemic lupus erythematosus during the past 26 years. Clin Rheumatol. 2014;33:57-63.

9. Zonana-Nacach A, Yañez P, Jiménez-Balderas FJ, Camargo-Coronel A. Disease activity, damage and survival in Mexican patients with acute severe systemic lupus erythematosus. Lupus. 2007;16:997-1000.

10. Namendys-Silva SA, Baltazar-Torres JA, Rivero-Sigarroa E, Fonseca-Lazcano JA, Montiel-López L, Domínguez-Cherit G. Prognostic factors in patients with systemic lupus erythematosus admitted to intensive care unit. Lupus. 2009;18:1252-1258.

11. Dumas G, Géri G, Montlahuc C, Chemam S, Dangers L, Brechot N, et al. Outcomes in critically ill patients with systemic rheumatic disease: a multicenter study. Chest. 2015;148:927-935

12. Quintero OL, Rojas-Villarraga A, Mantilla RD, Anaya JM. Autoimmune diseases in the intensive care unit. An update. Autoimmun Rev. 2013; 12:380-395

13. Siripaitoon B. Lertwises $S$. Uea-areewongsa $P$, et al. A study of Thai patients with systemic lupus erythematosus in the medical intensive care unit: epidemiology and predictors of mortality. Lupus. 2015;24:98-106.

14. Mak A, Cheung M, Chiew H, Liu Y, Ho RC. Global trend of survival and damage of systemic lupus erythematous: meta-analisis and meta-regression of observational studies from the 1950s to 2000s. Semin Arthritis Rheum. 2012;41:830-839.

15. Camargo JF, Tobón GJ, Fonseca N, Diaz JL, Uribe M, Molina F, et al. Autoimmune rheumatic disease in the intensive care unit: experience from a tertiary referral hospital and review of the literature. Lupus. 2005; 14:315-320.

16. Knaus WA, Draper EA, Wagner DP, Zimmerman JE. APACHE II: A severity of disease classification system. Crit Care Med. 1985;13: 818-829.

17. Vincent JL, Moreno R, Takala J, Willatts S, De-Mendonça A, Bruining H, et al. The SOFA (Sepsis-related Organ Failure Assessment) score to describe organ dysfunction/failure. On behalf of the Working Group on Sepsis-Related Problems of the European Society of Intensive Care Medicine. Intensive Care Med. 1996;22:707-710.

18. Ferreira FL, Bota DP, Bross A, Mélot C, Vincent, JL. Serial evaluation of the SOFA score to predict outcome in critically ill patients. JAMA. 2001;286:1754-1758.

19. ARDS Definition Task Force, Ranieri VM, Rubenfeld GD, Thompson BT, Ferguson ND, Caldwell E, et al. Acute respiratory distress syndrome: the Berlin Definition. JAMA. 2012;307:2526-2533.

20. Hess DR. Respiratory mechanics in mechanically ventilated patients. Respir Care. 2014;11:1773-1794.

21. Hsu CL, Chen KY, Yeh PS, Hsu YL, Chang HT, Shau WY, et al. Outcome and prognostic factors in critically ill patients with systemic lupus erythematosus: a retrospective study. Crit Care. 2005;9:R177-R183.

22. Kuroda Y. Neurocritical care update. J Intensive Care. 2016;4:36.

23. Iwashyna TJ, Ely EW, Smith DM, Langa KM. Long-term cognitive impairment and functional disability among survivors of severe sepsis. JAMA. 2010;304:1787-1794.

24. Bhattacharyya S, Helfgott SM. Neurologic complications of systemic lupus erythematosus, Sjögren syndrome and rheumatoid arthritis. Semin Neurol. 2014;34:425-436.

25. Haas SA, Lange T, Saugel B, Petzoldt M, Fuhrmann V, Metschke M, et al. Severe hyperlactatemia, lactate clearance and mortality in unselected critically ill patients. Intensive Care Med. 2016;42:202-210.

26. Juneja D, Singh O, Dang R. Admission hyperlactatemia: causes, incidence, and impact on outcome of patients admitted in general medical intensive care unit. J Crit Care. 2011;26:316-320. 